\title{
Study of Automobile SBC on Varied Pavement with Fuzzy Slide Model Adaptive Control
}

\author{
Renyun Sun ${ }^{\star}$, Wenhao Yang, Xiaowei Wang and Jinghong Zhong \\ School of Automobile and Transportation, Xihua University, Chengdu, 610039, China \\ ${ }^{*}$ Corresponding author
}

Keywords: Automobile SBC, Vary pavement, Fuzzy control, Slide mode control, Adaptive control.

\begin{abstract}
The method that Fuzzy Slide Mode Adaptive Control (FSMAC) was applied to the motor vehicle Sensotronic Brake Control (SBC) system on vary pavement was studied. According to the fuzzy base-function extension form of the broad sense T-S fuzzy system, the fuzzy base-function network controller and two kind parameter adaptive laws of the automobile SBC were designed. The quasi-slide area or fuzzy slide model plane was determined by combining the fuzzy control with the slide mode vary structure. One of laws was selected to control by the error arriving border and it is inside and outside. The computer simulation was fulfilled for this system by using Matlab/Simulink software on one kind of certain pavement and several vary pavments. The results show that this has better adaptive ability for vary pavements, and its braking was smooth. And there are short braking distance and time. Those can efficaciously improve the braking performance.
\end{abstract}

\section{Introduction}

As a new electronic control braking system, the motor vehicle Sensotronic Brake Control (SBC) system has been developed to improve automobile braking performance in recent years.Compared with traditional braking system, there are increased response speed, decreased braking delay time and fault, short braking distance and improved braking compatibility among wheels. Traffic safety and controllability and stability are improved. With direct slip ratio control, the motor vehicle SBC system makes braking performance,suitability and robustness better on vary pavements.And this receives the best effect of peak adhesion coefficient on current pavement ${ }^{[1][2]}$. The method that Fuzzy Slide Mode Adaptive Control (FSMAC) was applied to study the motor vehicle Sensotronic Brake Control(SBC)system on vary pavements.

Based on the best slip ratio control between the wheels and the pavement, longitudinal peak adhesion coefficient $\mu_{x i}^{*}$ is the controlled goal. In view of real-time control for different pavements, the control block diagram is shown in Fig.1. Some notations are defined as follows:

$s_{i o p}^{*}$ : the best slip ratio of current pavement;

$S_{x}$ : estimated practical slip, it was calculated according to the model in the context;

$\mu_{x}$ : longitudinal adhesion coefficient of wheels, which is the function of the wheel longitudinal slip ratio;

$p_{u}$ : hydraulic parameter output from controller;

$P$ : hydraulic pressure of braking wheel cylinder;

$T_{s b}$ : braking moment of the brake, they are time functions.

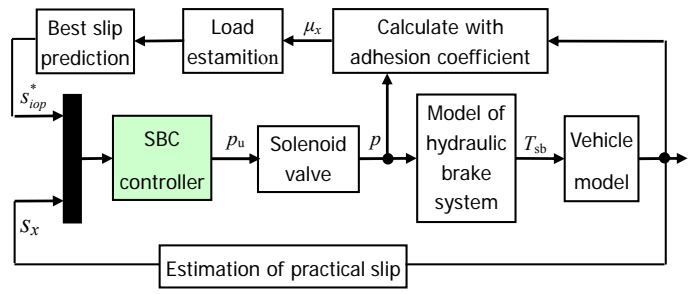

Figure 1. Control block diagrams based on optimum slip 


\section{Description of Automobile SBC System}

Automobile SBC system can be described as a single-input and single-output dynamic system in quarter vehicle model. According to the dynamical equation of a quarter vehicle model without rolling resistance, longitudinal additional force and air friction,the function of wheel slip ratio and brake moment can be received ${ }^{[2]}$ :

$$
\dot{s}_{x}=\left(\mu_{x}\left(s_{x}\right) g s_{x}-2 \mu_{x}\left(s_{x}\right) g+\frac{r_{r}}{J_{s r}} T_{s b}(t)\right) / u_{x}
$$

Where $s_{x}$ is estimated wheel longitudinal slip ratio; $\mu_{x}\left(s_{x}\right)$ is wheel longitudinal adhesion coefficient; $T_{s b}(t)$ is brake moment from the brake; $r_{r}$ is wheel woking redius; In the process of automobile driving and braking, this parameter may change in a certain range; It can be written as $r_{r}(t)$. Pretending it's invariable in this paper; $g$ is the acceleration of gravity; And $u_{x}$ is the running speed of motor vehicle.

With twice derivation of Eq.(1), the relationship between wheel longitudinal slip ratio $s_{x}$ and brake moment $s_{x}$ can be described as Eq.(2):

$$
s_{x}^{(3)}=f\left(s_{x}, \dot{s}_{x}, \ddot{s}_{x}\right)+g\left(\dot{T}_{s b}, \ddot{T}_{s b}\right)
$$

In this equation, $f$ and $g$ are bounded but unknown continuous function.

Using the brake line inlet pressure(outlet pressure of pressure regulator) $p(t)$ as input and the brake dynamic torque as output, comprehensive transfer function between the pipeline and the brake can be displayed as follows:

$$
\ddot{T}_{s b}(t)+2 \xi \omega_{n} \dot{T}_{s b}(t)+\omega_{n}^{2}=\frac{1}{2} \pi d_{w c}^{2} \mu_{b} R_{m} \omega_{n}^{2} p(t-\tau)
$$

Where, $\mu_{b}$ is coefficient of brake lining friction; $d_{w c}$ is the piston diameter of wheel braking cylinder; $\tau$ is a constant of delay time, trandational braking system is in the range of 20 to $50 \mathrm{~ms}$; $R_{m}$ is the average effective friction radius of the braking disc.

Based on Eq.(2) and Eq.(3), the equation is turned as Eq.(4):

$$
\left\{\begin{array}{l}
s_{x}^{(3)}=f\left(s_{x}, \dot{s}_{x}, \ddot{s}_{x}\right)+b p(t) \\
y=s_{x}
\end{array}\right.
$$

Where $b$ is unknown but bounded constant; $p(t)$ is system input, real number; $y$ is output,wheel slip ratio,real number;Eq.(4) is the controlled object of autobile SBC controller. It is a typical single-input and single-output system. This is useful to study and analyze the motor vehicle SBC system with Fuzzy Slide Mode Adaptive Control (FSMAC) method.

\section{The Approach of Determing Optimal Slip}

[2] provides 8 kinds of road peak adhesion coefficient, if we know the value of their corresponding slip, we can adopt the corresponding control strategy, search simultaneously, eventually reach the peak adhesion coefficient (ie the optimal slip), and the corresponding control is implemented. According to the [9] which provides the test data of 6 kind road adhesion coefficient and optimal slip, the data which no road is corresponding to are determined in accordance with the principle of taking the low value, conbined with 8 kinds of road conditions, subdivision is made, and the final 9 intervals of road parameters are obtained, which are taken as the actual control parameters. These peak adhesion coefficient and slip (optimal slip) of 9 kind road surface are shown in table1. The 
subscript $\mathrm{x}$ of each peak friction coefficient $\mu_{x i}^{*}$, adhesion coefficient $\mu_{x i}$ range estimated optimal slip range $\hat{s}_{x i}^{*}$, optimal slip predicted $s_{x i}^{*}$ shown in Table 1 indicate the longitudinal direction of vehicle, $i$ indicates the types of road surface $(i=1,2,3, \ldots \ldots)$.

The current road adhesion coefficient estimated by vehicle dynamic model is called " the current used adhesion coefficient"[9]. According to the adhesion coefficient range in table 1 which the current used adhesion coefficient falls into, the current road surface type and optimal slip are determined, and the control is carried out according to current optimal slip.

Table 1. The section of peak adhesion coefficient and optimal slip for control use

\begin{tabular}{|c|c|c|c|c|c|c|}
\hline Serial numberi & Road surface & Subdivision & $\mu_{x i}^{*}$ & $\mu_{x i}$ & $\hat{S}_{x i}^{*}$ & $S_{x i}^{*}$ \\
\hline 1 & ice & & 0.10 & $0.10>\mu$ & $0.10-0.20$ & \multirow{3}{*}{0.16} \\
\hline 2 & & blank0 & 0.15 & $0.20>\mu \geq 0.10$ & & \\
\hline 3 & snow & & 0.24 & $0.28>\mu \geq 0.20$ & $0.10-0.20$ & \\
\hline 4 & & blank1 & 0.45 & $0.62>\mu \geq 0.28$ & & 0.16 \\
\hline 5 & \multirow{4}{*}{$\begin{array}{l}\text { wet concrete, } \\
\text { wet gravel, } \\
\text { wet asphalt }\end{array}$} & Subdivision0 & 0.69 & $0.75>\mu \geq 0.62$ & $0.15-0.25$ & \multirow{4}{*}{0.20} \\
\hline 6 & & Subdivision 1 & 0.77 & $0.78>\mu \geq 0.75$ & $0.15-0.25$ & \\
\hline 7 & & Subdivision2 & 0.79 & $0.80>\mu \geq 0.78$ & $0.15-0.25$ & \\
\hline 8 & & Subdivision3 & 0.83 & $0.80>\mu \geq 0.78$ & $0.15-0.25$ & \\
\hline 9 & $\begin{array}{l}\text { dry asphalt, } \\
\text { dry concrete }\end{array}$ & & 0.92 & $0.98>\mu \geq 0.85$ & $0.14-0.20$ & 0.17 \\
\hline
\end{tabular}

As a result of the road surface adhesion coefficient estimated changes along with the slip change, even if in the high adhesion coefficient road surface, in the case of small slip, the adhesion coefficient estimated is still very small, therefore, the control is inevitably carried out according to the 1st kind road surface(ice)'s optimal slip. Suppose that the peak adhesion coefficient on a certain road surface between tire and road is bigger than the adhesion coefficient of any working codition and the peak adhesion coefficient does not appear in the time which wheel locks. As the change of brake pressure, the actual slip increases or decreases, when the current used adhesion coefficient estimated surpasses the $i^{\text {th }}$ peak adhesion coefficient but is smaller than the $i+1^{\text {th }}$, the region which arrow indicates is shown in fig. 2 , use $i+1^{\text {th }}$ road surface's optimal slip to implement the next step control, namely, regardless the current used adhesion coefficient vares from large to small or from small to large, takes the optimal slip of the road surface between the neighboring 2 kind road which the current estimated adhesion coefficient falls into whose peak adhesion coefficient is higher as the current optimal slip for control.

The mathematic expression of model-based current used adhesion coefficient (longitudinal) $\mu\left(s_{x}\right)$ is shown as below:

$$
\mu\left(s_{x}\right)=\frac{J_{s r} \dot{\omega}_{s r}+T_{s b}}{F_{s z} r_{r}}
$$

Where, $\dot{\omega}_{s r}$-wheel angular deceleration during braking;

$T_{s b}$ - brake torque;

$J_{s r}$ - moment of inertia of the wheel;

$F_{s z}$-normal reaction force road exerting on the wheels;

$r_{r}$-wheel radius.

The approach of determing the optimal slip, if we can get road types of optimal slip more, the smaller interval difference between the peak adhesion coefficient is smaller, the performance of wheel anti-lock is the better, and higher control accuracy and shorter braking distance can be obtained. However, due to its sensitiveness, it is easy to change, and the stability, anti-interference capability will be worse. Therefore, selecting types of road surface has influnence on the control 
system performance to a certain extent, but it must contain the minimum adhesion coefficient and maximum adhesion coefficient.

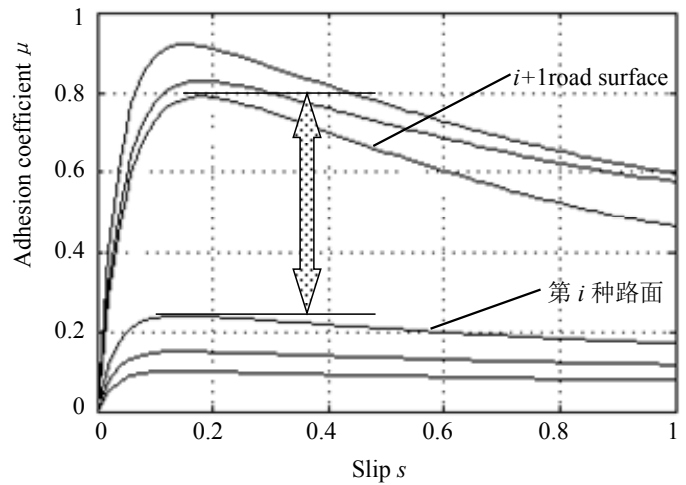

Figure 2. $\mu-s$ cuve on different road surface

\section{Design of FSMAC Controller}

There is necessarily external interference in the actual system.Leading with interference, the automobile SBC system is Eq.(5):

$$
\left\{\begin{array}{l}
s_{x}{ }^{(3)}=f\left(s_{x}, s_{x}{ }^{(1)}, s_{x}{ }^{(2)}\right)+b p(t)+d(t) \\
y=s_{x}
\end{array}\right.
$$

Where, $f$ is unknown but bounded continuous function; $b$ is unknown but bounded constant; $d(t)$ is unknown but bounded external interference; $u \in R, y \in R$ are the input and the output of the system.

$\boldsymbol{x}=\left(s_{x}, s_{x}{ }^{(1)}, s_{x}{ }^{(2)}\right)^{\mathrm{T}}=\left(s_{x 1}, s_{x 2}, s_{x 3}\right)^{\mathrm{T}}$ is hypothe- sized as the state vector of the system. They can be received by measurement. If function $f(\boldsymbol{x})$ and controller gain $b$ are well known. And $d(t)=0$ in Eq.(5). An ideal controller is designed as Eq.(6):

$$
\hat{p}=\frac{1}{b}\left[-f(\boldsymbol{x})+\beta S+\sum_{i=1}^{2} c_{i} e_{i+1}+y_{\text {ref }}^{(3)}\right]
$$

Where, $\beta$ is a positive constant. The selection of it's value should meet the multinomial: $h(u)=u^{3}+\left(\beta+c_{2}\right) u^{2}+\left(\beta c_{2}+c_{1}\right) u+c_{1}=0$ And it makes all roots located in the left-half plane.

It's very hard to satisfy the hypothesis in engineering design. So the control of ideal controller Eq.(6) is difficult to realize. But fuzzy base-function network controller can be used to approximate to the ideal controller $\hat{p}$.

According to the characteristics that broad sense T-S fuzzy system can use any precision approximation as controlled object, and we use Gaussian function as the membership function. The fuzzy base-function network controller for designing fuzzy logic system is Eq.(7) ${ }^{[4]}$ :

$$
p_{f z}(\boldsymbol{x} \mid \boldsymbol{\alpha})=\boldsymbol{\varphi}^{\mathrm{T}}(\boldsymbol{x}) \boldsymbol{\alpha}=\boldsymbol{\alpha}^{\mathrm{T}} \boldsymbol{\varphi}(\boldsymbol{x})
$$

The parameter adaptive law is as follows:

$$
\dot{\boldsymbol{\alpha}}=-\eta \boldsymbol{\varphi}(\boldsymbol{x}) \boldsymbol{c} \boldsymbol{e}=-\eta \cdot S \cdot \boldsymbol{\varphi}(\boldsymbol{x})
$$

Where $\boldsymbol{\varphi}=\left(\varphi_{1}, \varphi_{2}, \cdots, \varphi_{n}\right)^{\mathrm{T}}$ is fuzzy base-function vector. The fuzzy base-function of the broad sense T-S fuzzy system is Eq.(9) $)^{[4][7]}$ :

$$
\varphi_{i}(\boldsymbol{x})=\prod_{j=1}^{3} \mu_{A_{j}^{i}}\left(x_{j}\right) / \sum_{i=1}^{n}\left(\prod_{j=1}^{3} \mu_{A_{j}^{i}}\left(x_{j}\right)\right)
$$


$(i=1,2, \cdots, n)$

Where, $\sum_{i=1}^{n} \varphi_{i}(x)=1$ and $0 \leq \varphi_{i}(x) \leq 1$. For each input vector $x, \varphi_{i}(x)$ is the ith weight of fuzzy rules. $\boldsymbol{\alpha}=\left(\alpha_{1}, \alpha_{2}, \cdots, \alpha_{n}\right)^{\mathrm{T}}$ is adjustable parameter vector. Every set corresponds one rule.

In order to improve the performance of fuzzy adaptive control system, a parameter adaptive law is used as follows:

$$
\begin{aligned}
\dot{\boldsymbol{\alpha}} & =-\eta \cdot p(\dot{S}, S) \cdot \boldsymbol{\varphi}(\boldsymbol{x}) \\
& =-\eta \cdot\left(\dot{S}+\beta_{d} S\right) \cdot \boldsymbol{\varphi}(\boldsymbol{x})
\end{aligned}
$$

Where $\beta_{d}$ is a positive constant. The control performance was improved. But convergence speed was slower.

The quasi-slide area or fuzzy slide model plane was designed by combining the fuzzy control with the slide mode vary structure ${ }^{[4]}$. Pretending one boundary layer thickness is $\theta$. There $\theta \geq 0$.It's boundary layer width is $\sigma=\theta / \beta$. The control method is as follows: (1) If the controlled object is out of the quasi-slide area, parameter adaptive law in Eq.(8) will be used.The error state will fast converge to the quasi-slide area. (2) If the controlled object is in the quasi-slide area, parameter adaptive law in Eq.(10) will be used. The control performance will be improved possibly. Convergence speed Converges to the best trace with exponential rule. Algebra distance of error state boundary layer is Eq.(11):

$$
S_{D}=S-\theta \cdot \text { area_judgefunction }\left(\frac{S}{\theta}\right)
$$

Where the judge function is defined as Eq.(12):

$$
\text { area_judgefunction }\left(\frac{S}{\theta}\right)= \begin{cases}+1 & \frac{S}{\theta}>1 \\ \frac{S}{\theta} & \left|\frac{S}{\theta}\right| \leq 1 \\ -1 & \frac{S}{\theta}<-1\end{cases}
$$

The characteristics of $S_{D}$ are shown as following: when $|S|>\theta,\left|S_{D}\right|=|S|-\theta$ and $\dot{S}_{D}=\dot{S}$; when $|S| \leq \theta, S_{D}=\dot{S}_{D}=0$. If the parameter self-adaption isn't adjusted, inertia turns back to the slide model plane.

Therefore, accordingly the ideal controller Eq.(6) has been altered to Eq.(13):

$$
\hat{p}=\frac{1}{b}\left[-f(\boldsymbol{x})+\beta S_{D}+\sum_{i=1}^{2} c_{i} e_{i+1}+y_{\text {ref }}^{(3)}\right]
$$

Error state is Eq.(14):

$$
\dot{S}+\beta S_{D}=0
$$

If $|S|>\theta$, when $t \rightarrow \infty, S_{D} \rightarrow 0,|S| \rightarrow \theta$. So, in the domain of $e(t) \rightarrow 0$, the magnitude of the domain has a connection with boundary layer thickness $\theta$.

If the fuzzy base-function network approximation controller is constant, Eq.(7) will be used as Eq.(15):

$$
p_{f z}(\boldsymbol{x} \mid \boldsymbol{\alpha})=\boldsymbol{\varphi}^{\mathrm{T}}(\boldsymbol{x}) \boldsymbol{\alpha}=\boldsymbol{\alpha}^{\mathrm{T}} \boldsymbol{\varphi}(\boldsymbol{x})
$$

Parameter adaptive law will be chosen as Eq.(16):

$$
\dot{\boldsymbol{\alpha}}=\eta \cdot S_{D}(t) \cdot \boldsymbol{\varphi}(\boldsymbol{x})
$$


Where $\eta>0$ is self-adapting ratio or learning rate of $\boldsymbol{\alpha}$.

In order to reducing the influence from approximation error and external interference, their balance controller $p_{\mathrm{c}}$ was inserted during the process of controller design. Balance controller is designed as Eq.(17):

$$
p_{c}= \begin{cases}I \cdot \operatorname{sgn}(S) & |\mathrm{S}|>\theta \\ \dot{S}+\beta_{d} S & |\mathrm{~S}| \leq \theta\end{cases}
$$

Where $\mathrm{I}=1, \beta_{d}>0, \operatorname{sgn}(\cdot)$ is sign function.

The ultimate closed loop controller design is as follows:

$$
p=p_{f z}(\boldsymbol{x} \mid \boldsymbol{\alpha})+k_{\mathrm{c}} p_{\mathrm{c}}
$$

Where $k_{\mathrm{c}}$ is a balance controller gain. The gain is made up of two parts: control gain $k_{\zeta}$ of fuzzy base-function network approximation error $\zeta$ and external interference suppressor gain $k_{d}$. And the relationship between them is shown in Eq.(19).

$$
k_{c}=k_{\varsigma}+k_{d}
$$

The values of $k_{\zeta}$ and $k_{d}$ are defined in Eq.(20) and Eq.(21):

$$
\begin{gathered}
\dot{k}_{\zeta}=\lambda\left|S_{D}\right| \\
k_{d}=\frac{D_{\text {max }}}{b_{\text {min }}}
\end{gathered}
$$

Where, $\lambda$ is the leaning rate of $k_{\zeta}, D_{\max }$ is the upperbound of external interference, $|d(t)| \leq D_{\max }, b_{\min }$ is the downbound of original system control gain, $0<b_{\min }<b$.

The equation of tracing error state is shown in Eq.(22):

$$
e^{(3)}=b(\hat{p}-p)-\beta S_{D}-\sum_{j=1}^{2} c_{j} e_{j+1}-d(t)
$$

\section{Simulation Test}

The function approximation ability of fuzzy base-function network controller is connected with the fuzzy rules of controller. If the description of fuzzy rules is fuller, the function approximation error is less. Otherwise, it is larger. Fuzzy base function network controller doesn't ask too much of the language model precision with the use of approximation error and external interference. The larger the value of $\beta_{d}$, the higher the convergence precision, and the faster the convergence speed. If $\beta_{d}$ is a constant value, it will ensure that the tracing error converges to the scheduled precision at a set speed.

The input parameters are motor wheel slip ratio and it's rate of change. The base universe of slip ratio is $[0.05,0.35]$. The base universe of slip ratio rate of change is $[-2,2]$. The universe is divided into 5 levels. Their language values are very small, less, moderate, larger, great, and NL, NS, ZE, PS, PL. Gaussian function is chosen as the membership function. The function expression is as follows:

$$
\mu_{A_{1}^{J}}=\exp \left[-\left(\frac{s_{x}-0.05+0.075(1-j)}{0.03186}\right)^{2}\right]
$$




$$
\mu_{A_{2}^{\prime}}=\exp \left[-\left(\frac{s_{x c}+j-3}{0.4247}\right)^{2}\right]
$$

According to those, the polynomial expression of tracing error is defined as Eq.(25).

$$
S=\ddot{e}+c_{2} \dot{e}+c_{1} e
$$

Because the speed has effect on peak adhesion coefficient and the best slip ratio. The relationship between the speed $v$ and the peak adhesion coefficient $\mu_{x i o p}^{*}$ and the relationship between the speed $v$ and the best slide ratio $s_{i o p}^{*}$ on defferent pavements are shown as follows:

$$
\begin{gathered}
\mu_{\text {xiop }}^{*}=\bar{\mu}_{\text {xiop }}^{*}+0.002 \times(64.0-v) \\
s_{\text {iop }}^{*}=\bar{s}_{\text {iop }}^{*}+0.165 \times \lg (64.0 / v)
\end{gathered}
$$

Where, $v$ is vehicle speed, $\bar{\mu}_{x i o p}^{*}$ and $\bar{s}_{i o p}^{*}$ are estimated adhesion coefficient and slip. During the application of the last two equaltion, $v$ can't $0, v_{\min }$ is $10 \mathrm{~km} / \mathrm{h}$. If it is lower than the value.v is $10 \mathrm{~km} / \mathrm{h}$. The brake initial speed $v=160 \mathrm{~km} / \mathrm{h}(44.44 \mathrm{~m} / \mathrm{s})$. The method that the best slip ratio searched by successive for pavement was used with estimated adhesion coefficient ${ }^{[1]}$. With the simulation between S-function written in Matlab and Simulink, the block diagram of simulation is shown in Fig.3.

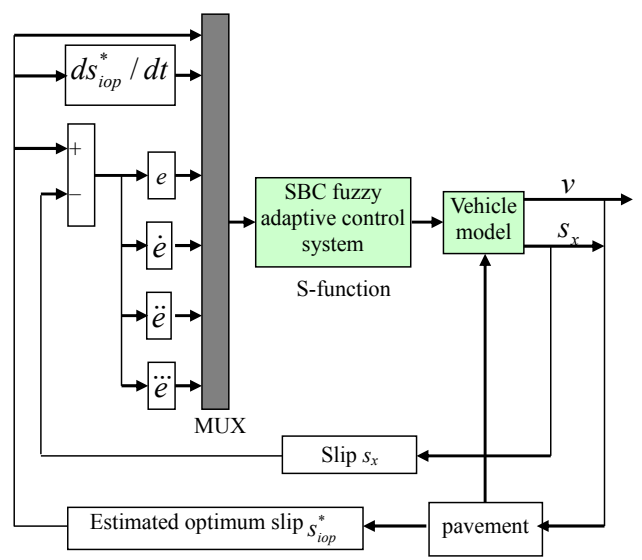

Figure 3. Motor vehicle SBC FSMAC system structure

Choice parameters: $\eta=800, \theta=0.1, \quad \beta_{d}=800, \lambda=0.1, D_{\max }=0.1, \quad b_{\min }=0.0369, \quad c_{1}=1.6 e+4$, $c_{2}=160$.

\section{Simulation 1}

Brake on certain pavement $(\mu=0.86)$.Curves about wheel and body speeds, trancing ratio and so on are shown as Fig.4, Fig.5, Fig.6.

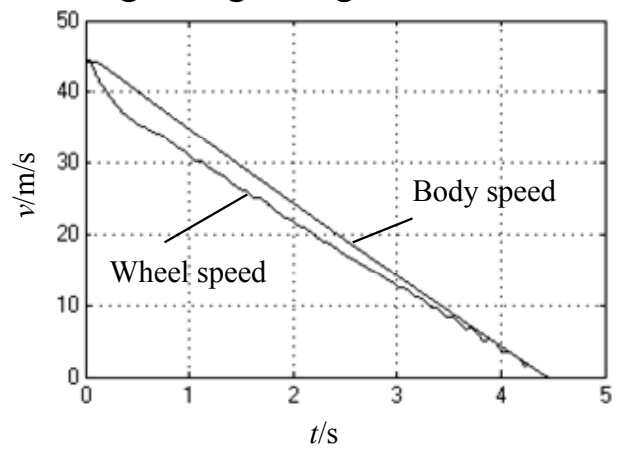

Figure 4. Wheel and body speed curve

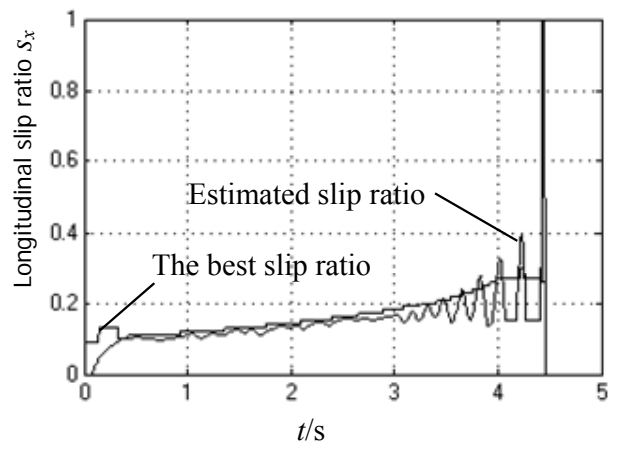

Figure 5. Estimated slip compare with optimum slip 


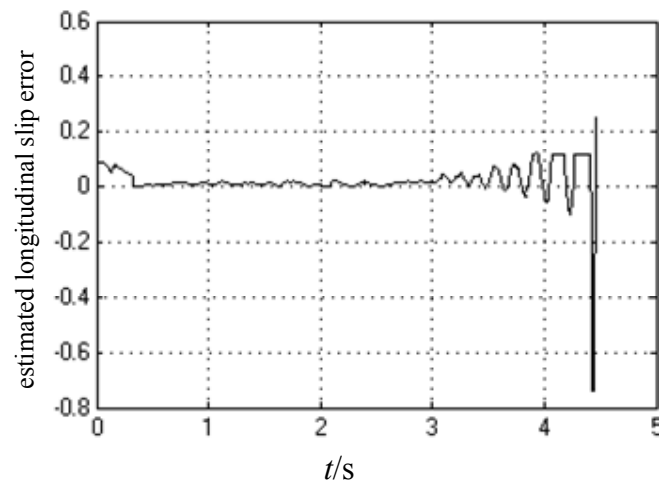

Figure 6. Estimated slip error

Brake distance is about $99.45 \mathrm{~m}$. Brake time is $4.455 \mathrm{~s}$. The range of error is $-0.71 \sim 0.23$.

\section{Simulation 2}

Brake on vary pavements. The input single from vary pavement for simulation is shown as Fig.7. Now, curves about wheel and body speeds, trancing about slip and so on are shown in Fig.8, Fig.9, Fig.10, Fig.11.

Brake distance is about $124.5 \mathrm{~m}$. Brake time is $7.2 \mathrm{~s}$. The range of error is $-0.71 \sim 0.23$.

The result of the simulation is that, with the method of Fuzzy Slide Mode Adaptive Control (FSMAC), peak adhesion coefficient is basically guaranteed to close to the brake whether on vary pavement or certain. The use of ground adhesion coefficient is better. And the results of speed, estimated adhetion coefficient and estimated slip ratio are smooth during the process of brake. These show that the change in the control process is steady.

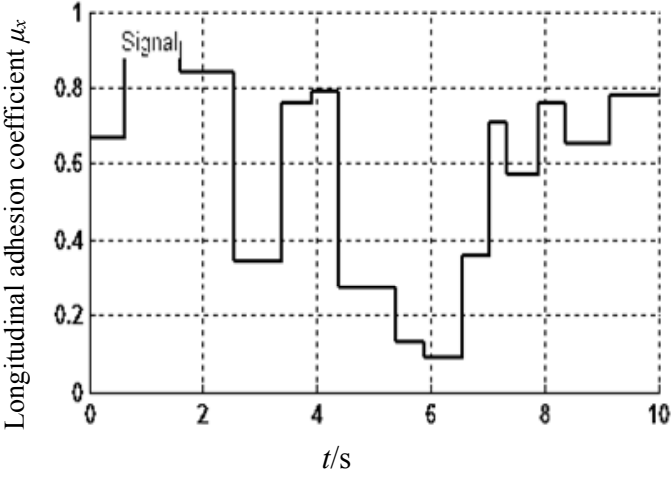

Figure 7. Input single on vary pavement

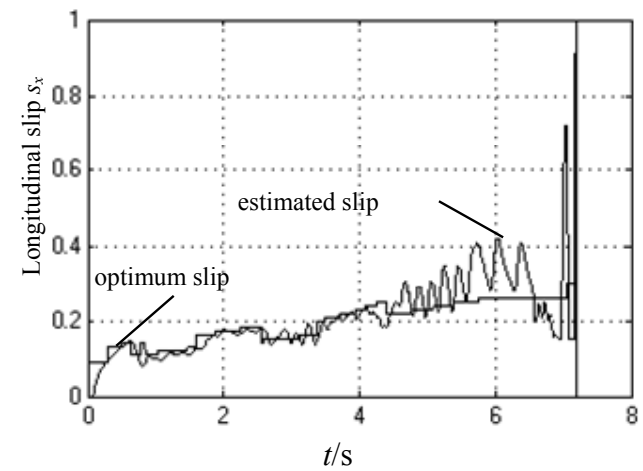

Figure 9. Estimated slip compare with optimum slip

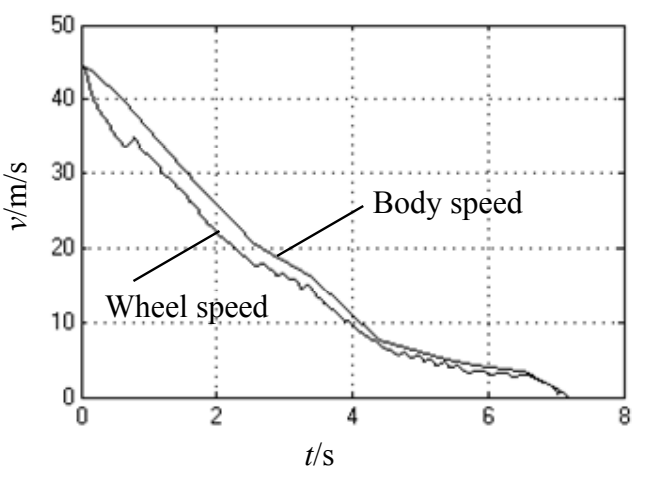

Figure 8 . Wheel and body speed curves

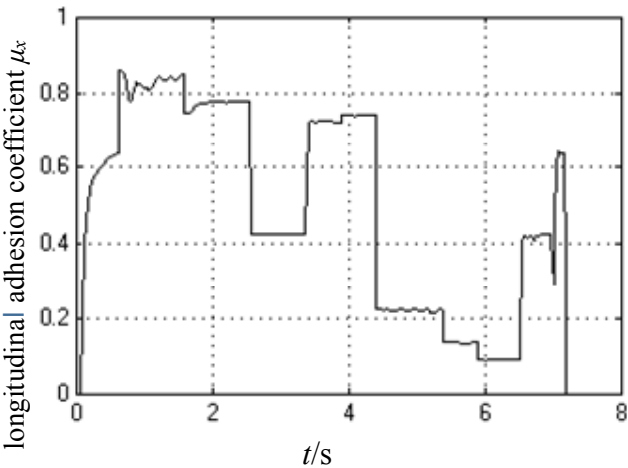

Figure 10. Estimated adhesion coefficient 


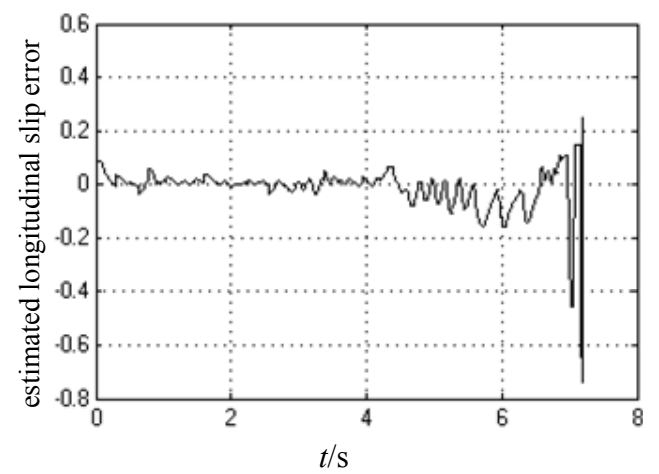

Figure 11. Estimated slip error

\section{Analysis and Discussion}

(1) The errors of the best slip and estimated slip are basically in the range of \pm 0.1 , very smaller. Big error appears at lower speed. Now the dangerous is impossible during the process of brake.There is no wheel lock in the whole brake process. This has a good effect on improving vehicle brake stability.

(2) The fluctuation of wheel speed exits at lower speed in the whole brake process. Through simulation, I found that it is difficult to reduce this fluctuation.

(3) From simulation on vary pavement, we can find that the change of the optimum slip is caused by the change of vehicle speed or pavement.From Fig.4 and Fig.8, the tracing from estimated slip to the optimum slip is better.

(4) FSMAC needs many definite parameters. $\eta, \theta, \beta_{d}, \lambda, c_{1}, c_{2}$ and so on.Some are convenient to determine, and some are difficult. So FSMAC can't completely realize the optimal control. If the parameters are appropriate, and the best combination effect can be received.And the ideal controlled result can be received, too.

(5) The peak adhesion coefficience changes due to the change of wheel speed or pavement. Comparative analysis from Fig. 8 and Fig.10 shows that the tracing from estimated adhesion coefficient to peak adhesion coefficient is better. And it can acquire the shorten brake distance and brake time.

(6) Because the speed has effect on peak adhesion coefficient and the best slip ratio. The method that the best slip ratio searched by successive for pavement has better tracing performance for the change of speed in Fig.9.

(7) Combining Fig.7 with Fig.8, we can find that the change of pavement has effect on variation tendency of wheel speed and body speed. The wheel speed and body speed descend faster on high adhesion pavement, and the curve is rapid; On low adhesion pavement, it is slower and gentle.If the two adjacent pavement adhesion conditions have great changes, the speeds of the wheel and the body will also have gteat changes. This is consistent with practical situation. And no wheel lock phenomenon is required now.

(8) The worse tracing performance at lower speed is connected with controller. It can be improved by changing performance parameters of controller. Wheel lock is allowed this time (when speed is lower than $10 \mathrm{~km} / \mathrm{h}$ ). And the controlling logic of wheel lock can be used directly when it is lower than a certain speed.

\section{Conclusion}

(1) The technique of fuzzy slide model adaptive control was used in this paper. The method that the optimum slip is determined by searching step by step for every pavement can solve the issue of the optimum slip control of vehicle SBC on vary pavements. 
(2) The vary pavement studied in this paper is close to the real road condition. The result is valuable for developing the vehicle practical SBC with fuzzy adaptive controller.

\section{References}

[1] SUN Renyun, LI Zhi. Study on SBC Optimum Slip Based on Variable Pavement[J], China Mechanical Engineering, 2008.03, 19(4):481-486.

[2] YU Zhisheng. Theory of Automobile[M] (third edition). Beijing: Machinery Industry Press.2000:75, 97-98.

[3] CHEN Hongwei. Research on Anti-lock Braking Dynamic Response Analysis of Disc Brake[D].Doctoral Dissertation of Jilin University, 1999.6.

[4] ZHANG Huaguang. Theory of Adaptive Fuzzy Control and Application[M], Beijing University of Aeronautics and Astronautics Press, 2002.7

[5] Sungchul Jee, Yoram Koren, Adaptive fuzzy logic controller for feed drives of a CNC machine tool[J], Mechatronics, 2004, 14:299-326

[6] Shaocheng Tong, Han-Xiong Li, Wei Wang, Observer-based adaptive fuzzy control for SISO nonlinear systems[J], Fuzzy Sets and Systems,2004, 148:355-376

[7] Chen-Sheng Ting, Stability analysis and design of Takagi-Sugeno fuzzy systems[J], Information Sciences, 2006, 176: 2817-2845

[8] Yong-Tae Kim, Z.Zenn Bien, Robust adaptive fuzzy control in the presence of external disturbance and approximation error [J], Fuzzy Sets and Systems,2004,148:377-393.

[9] Liu Zhaodu. Mathematical models of tire longitudinal road adhesion and their use in the study of road vehicle dynamics[J]. Journal of Beijing Institute of Technology. 1996, Vol.5(2):192-204.

[10]XUE Dingyu. Computer Aided Design for Control System -MATLAB Language and Application $[\mathrm{M}]$ (second edition), Tsinghua University Press, 2006.3. 